\title{
Diagnosis by a Waiter and a Mars Explorer
}

\author{
Nando de Freitas ${ }^{\star \dagger}$, Richard Dearden ${ }^{\ddagger}$, Frank Hutter ${ }^{\S}$, Rubén Morales-Menéndez ${ }^{\dagger}$, Jim Mutch ${ }^{\dagger}$, and David Poole ${ }^{\dagger}$
}

(Invited Paper)

\begin{abstract}
This paper shows how state-of-the-art state estimation techniques can be used to provide efficient solutions to the difficult problem of real-time diagnosis in mobile robots. The power of the adopted estimation techniques resides in our ability to combine particle filters with classical algorithms, such as Kalman filters. We demonstrate these techniques in two scenarios: a mobile waiter robot and planetary rovers designed by NASA for Mars exploration.
\end{abstract}

Index Terms-State estimation, robotics, diagnosis, RaoBlackwellised particle filtering.

\section{INTRODUCTION}

FAULT diagnosis is a critical task for the autonomous operation of mobile robots, such as planetary rovers. The objective of diagnosis systems is to detect the discrete state of operation of a robot given a stream of observations [1], [2], [3], [4]. For example, given measurements of currents and images of the environment, a robot might need to automatically diagnose whether any of its wheels are stuck. In this example, the discrete states could be left wheel stuck and right wheel stuck. Once the robot knows its discrete state, it can generate a control action to solve its current problem.

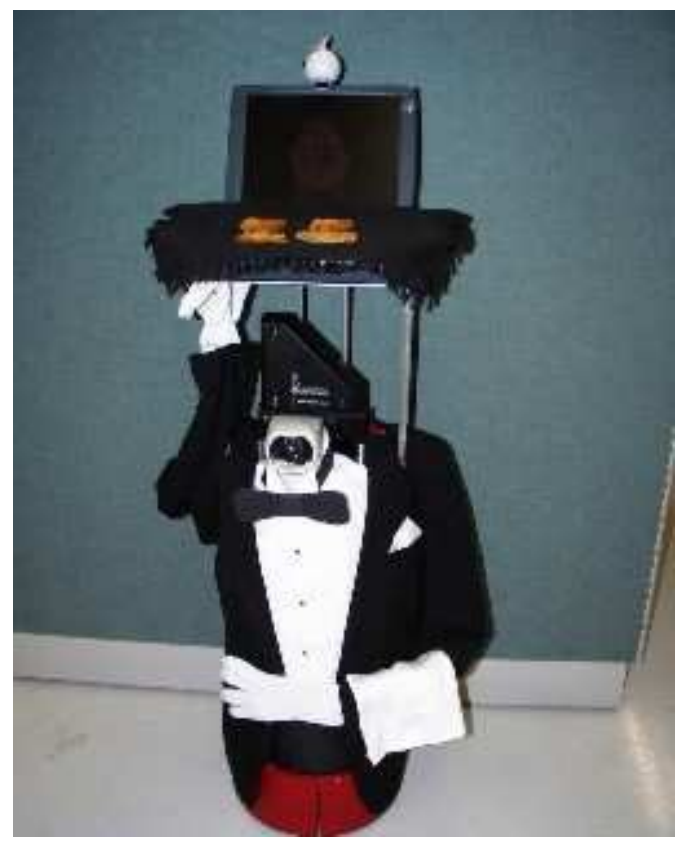

Fig. 1. Jos'e, winner of the 2001 AAAI Hors d'Oeuvres Competition [5].

\footnotetext{
* Authorship in alphabetical order.

$\dagger$ University of British Columbia

\# NASA Ames

$\S$ Darmstadt University of Technology
}

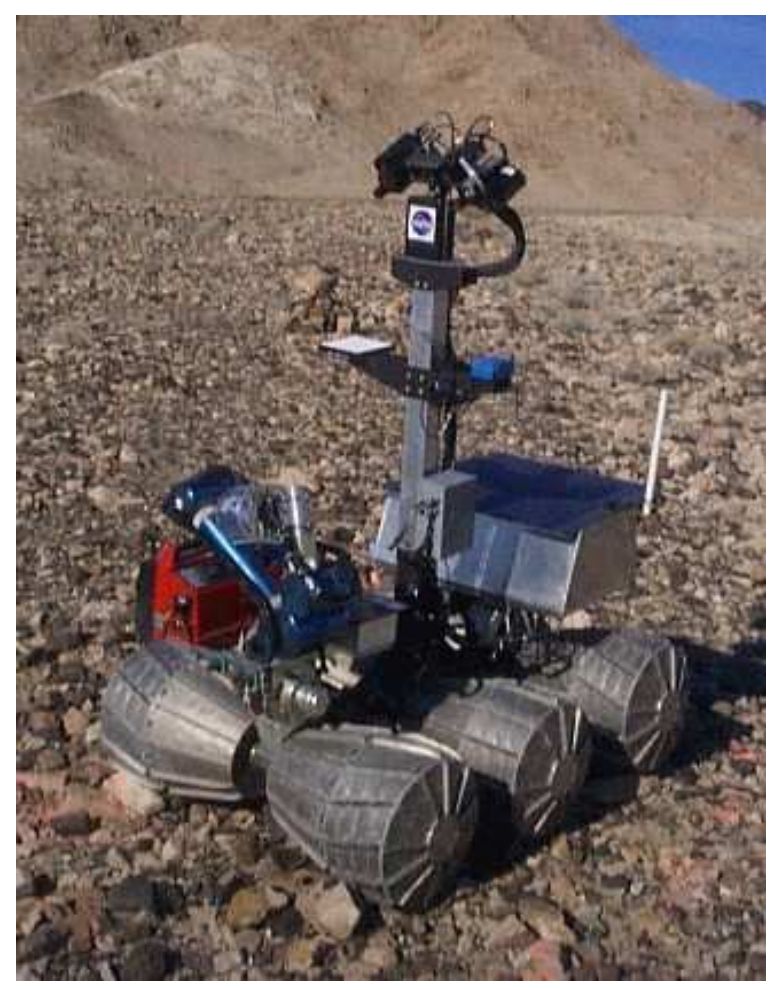

Fig. 2. The Marsokhod planetary rover.

This problem is hard because the discrete state of the robot depends on context. A high current when going uphill or over an obstacle may be normal, but the same current on a flat surface could indicate a fault. Hence, in order to obtain good estimates of the discrete states of the robots, we also need to infer the many dynamic, continuous states. This problem results in models that are intractable. Fortunately, particle filters can be used to provide accurate approximations.

Particle filters go back to the first publically available paper in the modern field of Monte Carlo simulation [6]; see [7] for a comprehensive review. One of the major drawbacks of PF is that sampling in high-dimensional spaces can be inefficient. In some cases, however, the model has "tractable substructure", which can be analytically marginalized out, conditional on certain other nodes being imputed. The analytical marginalization can be carried out using standard algorithms, such as the Kalman filter, the HMM filter, the junction tree algorithm for general dynamic Bayesian networks (DBNs) [8], or, any other finite-dimensional optimal filters. The advantage of this strategy is that it can drastically reduce the size of the space over which we need to sample. 
Marginalizing out some of the variables is an example of the technique called Rao-Blackwellisation, because it is related to the Rao-Blackwell formula: see [9] for a general discussion. Rao-Blackwellised particle filters (RBPF) have been applied in specific contexts such as mixtures of Gaussians [10], [11], [12], fixed parameter estimation [13], HMMs [11], [12], Dirichlet process models [14], and DBNs [8]. An excellent theoretical treatment of the algorithms adopted in this paper is presented in [15].

In the paper, we also propose a version of RaoBlackwellised particle filtering that does one-step look-ahead to select good sampling regions. We show that the overhead of the extra processing per particle of this more sophisticated method is more than compensated by the decrease in error and variance.

We begin the paper by presenting the mathematical models and various particle filtering algorithms. Next, we demonstrate our algorithms for state estimation on three robots: a Real World Interfaces B-14 mobile robot with a B-12 base (see Figure 1), a K-9 planetary rover (Figure 15) and a Marsokhod planetary rover (Figure 2).

\section{Mathematical Model}

We represent the complex nonlinear process (robot system) with a dynamic mixture of linear processes. In addition to the continuous state variables corresponding to each linear process, we have a discrete state variable that determines the linear regime of operation. In the fault diagnosis setting, each regime corresponds to a particular fault. Different regimes could, however, be used to represent levels of the fault or different internal states of the robot for the purposes of controlling it automatically. We acquire data for each regime separately. This data enables us to do off-line identification with the EM algorithm [16], [17]. The models estimated with EM are validated on test set data to ensure that we have captured the behaviour of the process adequately. In addition, we ensure that the data generated by the models is statistically similar to the real data; see [18] for more details on how this is done.

Once the stationary parameters have been identified, realtime Rao-Blackwellised particle filtering (RBPF) algorithms are used to estimate the continuous and discrete states of the system on-line. These estimates are used to determine the type of fault and control policies.

In mathematical terms, we adopt the following state space representation ${ }^{1}$ :

$$
\begin{aligned}
z_{t} & \sim P\left(z_{t} \mid z_{0: t-1}, x_{0: t-1}, y_{1: t-1}, u_{1: t-1}\right) \\
x_{t} & =A\left(z_{t}\right) x_{t-1}+B\left(z_{t}\right) w_{t}+F\left(z_{t}\right) u_{t} \\
y_{t} & =C\left(z_{t}\right) x_{t}+D\left(z_{t}\right) v_{t}+G\left(z_{t}\right) u_{t},
\end{aligned}
$$

${ }^{1}$ NOTATION: For a generic vector $\theta$, we adopt the notation $\theta_{1: t} \triangleq$ $\left(\theta_{1}, \theta_{2}, \ldots, \theta_{t}\right)^{\prime}$ to denote all the entries of this vector at time $t$. For simplicity, we use $\theta_{t}$ to denote both the random variable and its realisation. Consequently, we express continuous probability distributions using $p\left(d \theta_{t}\right)$ instead of $\operatorname{Pr}\left(\theta_{t} \in d \theta_{t}\right)$ and discrete distributions using $p\left(\theta_{t}\right)$ instead of $\operatorname{Pr}\left(\theta_{t}=\theta_{t}\right)$. If these distributions admit densities with respect to an underlying measure $\mu$ (counting or Lebesgue), we denote these densities by $p\left(\theta_{t}\right)$. For example, when considering the space $\mathbb{R}^{n}$, we will use the Lebesgue measure, $\mu=d \theta_{t}$, so that $P\left(d \theta_{t}\right)=p\left(\theta_{t}\right) d \theta_{t}$. where,

- $y_{t} \in \mathbb{R}^{n_{y}}$ denotes the measurements.

- $x_{t} \in \mathbb{R}^{n_{x}}$ denotes the unknown continuous states.

- $u_{t} \in \mathcal{U}$ is a known control signal.

- $z_{t} \in\left\{1, \ldots, n_{z}\right\}$ denotes the unknown discrete states (normal operation and faulty conditions).

- The noise processes are i.i.d Gaussian: $w_{t} \sim \mathcal{N}(0, I)$ and $v_{t} \sim \mathcal{N}(0, I)$.

- The parameters $(A, B, C, D, F, G)$ are identified matrices with $D\left(z_{t}\right) D\left(z_{t}\right)^{\mathrm{T}}>0$ for any $z_{t}$. Typically, these matrices are estimated with the EM algorithm for linear dynamic systems [16]. That is, one simply fixes $z$ and obtains data from the physical process. With $z$ known, it is straightforward to estimate these matrices from the measured data using the EM algorithm. We have to repeat this process for each possible value of $z$.

- The initial states are $x_{0} \sim \mathcal{N}\left(\mu_{0}, \Sigma_{0}\right)$ and $z_{0} \sim p\left(z_{0}\right)$.

- The transition kernel $P\left(z_{t} \mid z_{0: t-1}, x_{0: t-1}, y_{1: t-1}\right)$ could correspond to either a discrete Markov transition matrix $P\left(z_{t} \mid z_{t-1}\right)$, a continuous transition density, a DBN with some independence structure that could be exploited, a hierarchical parameterised model, etc. The important thing is that we can easily derive particle filters using this very general expression for the kernel. In our experiments we adopted a Markov transition matrix. Our model is therefore a jump Markov linear Gaussian (JMLG) model.

For clarity of presentation, we state explicitly that our model implies the continuous densities:

$$
\begin{aligned}
p\left(x_{t} \mid z_{t}, x_{t-1}, u_{t}\right) & =\mathcal{N}\left(A\left(z_{t}\right) x_{t-1}+F\left(z_{t}\right) u_{t}, B\left(z_{t}\right) B\left(z_{t}\right)^{\mathrm{T}}\right) \\
p\left(y_{t} \mid x_{t}, z_{t}, u_{t}\right) & =\mathcal{N}\left(C\left(z_{t}\right) x_{t}+G\left(z_{t}\right) u_{t}, D\left(z_{t}\right) D\left(z_{t}\right)^{\mathrm{T}}\right) .
\end{aligned}
$$

For ease of presentation, we also drop the control signal $u$ from the argument of the various probability distributions. We should notice that for each realization of $z_{t}$, we have a single linear-Gaussian model. If we knew $z_{t}$, we could solve for $x_{t}$ exactly using the Kalman filter algorithm. The directed acyclic graphical model representation of our model is shown in Figure 3.

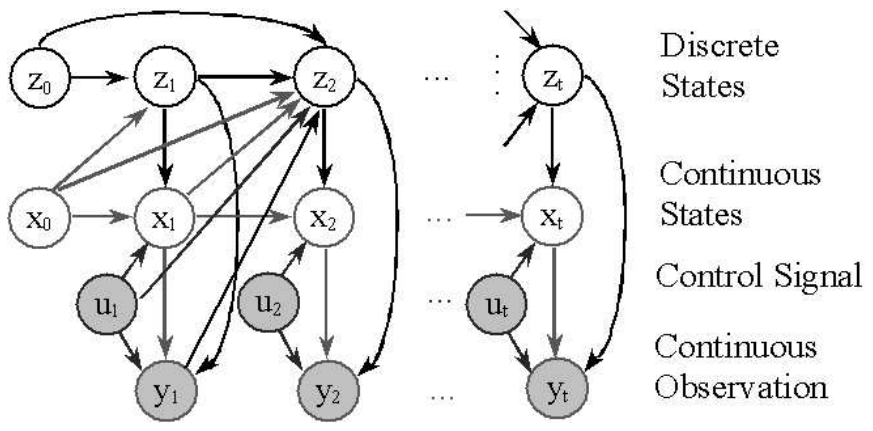

Fig. 3. Probabilistic graphical model. Note that by conditioning on $z$, we have a simple linear-Gaussian graphical model. 
The aim of the analysis is to compute the marginal posterior distribution of the discrete states $p\left(z_{0: t} \mid y_{1: t}\right)$. This distribution can be derived from the posterior distribution $p\left(d x_{0: t}, z_{0: t} \mid y_{1: t}\right)$ by standard marginalisation. The posterior density satisfies the following recursion

$$
\begin{aligned}
p\left(x_{0: t}, z_{0: t} \mid y_{1: t}\right)=p\left(x_{0: t-1}, z_{0: t-1} \mid y_{1: t-1}\right) \\
\times \frac{p\left(y_{t} \mid x_{t}, z_{t}\right) p\left(x_{t}, z_{t} \mid x_{0: t-1}, z_{0: t-1}, y_{1: t-1}\right)}{p\left(y_{t} \mid y_{1: t-1}\right)}
\end{aligned}
$$

This recursion involves intractable integrals in the denominator. One, therefore, has to resort to some form of numerical approximation scheme.

\section{PARTICle Filtering}

The idea of Monte Carlo simulation is to draw an i.i.d. set of samples $\left\{x^{(i)}\right\}_{i=1}^{N}$ from a target distribution $p(d x \mid y)$ defined on a high-dimensional space $\mathcal{X}$ (for simplicity, we are ignoring $z$ for the time being). These $N$ samples can be used to approximate the target density with the following empirical point-mass function

$$
\widehat{P}_{N}\left(d x_{0: t} \mid y_{1: t}\right)=\frac{1}{N} \sum_{i=1}^{N} \delta_{x_{0: t}^{(i)}}\left(d x_{0: t}\right)
$$

where $\delta_{x^{(i)}}(d x)$ denotes the delta-Dirac mass located at $x^{(i)}$. That is, we are simply approximating a complex distribution with a histogram of the samples as shown in Figure 4.

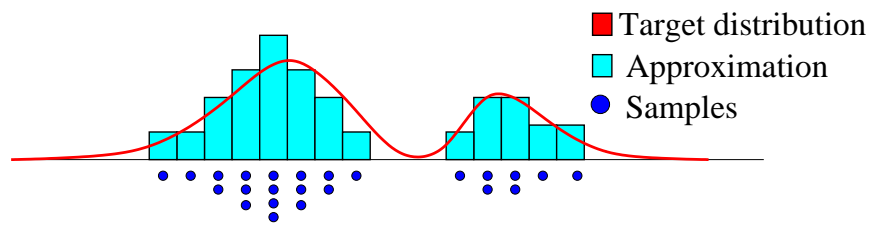

Fig. 4. Approximating the target distribution using a histogram of the Monte Carlo samples in a simple one-dimensional example.

Consequently, one can approximate any integrals (expectations such as the mean and variance of $x) I(f)$ with tractable sums $I_{N}(f)$ that converge as follows

$I_{N}(f)=\frac{1}{N} \sum_{i=1}^{N} f\left(x_{0: t}^{(i)}\right) \underset{N \rightarrow \infty}{\stackrel{a . s .}{\longrightarrow}} I(f)=\int f\left(x_{0: t}\right) p\left(d x_{0: t} \mid y_{1: t}\right)$

That is, the estimate $I_{N}(f)$ is unbiased and by the strong law of large numbers, it will almost surely (a.s.) converge to $I(f)$. From the set of random samples $\left\{x^{(i)} ; i=1, \ldots, N\right\}$, one can easily estimate any quantity $I(f)$ and the rate of convergence of this estimate is independent of the dimension of the integrand. In contrast, any deterministic numerical integration method has a rate of convergence decreasing as the dimension of the integrand increases. Unfortunately, it is usually impossible to sample efficiently from the posterior density $p\left(x_{0: t} \mid y_{1: t}\right)$ at any time $t, p\left(x_{0: t} \mid y_{1: t}\right)$ being multivariate, non standard and only known up to a proportionality constant. So we turn to importance sampling.
Let us introduce an arbitrary so-called importance sampling distribution (also often referred to as the proposal distribution or the importance function) $q\left(x_{0: t} \mid y_{1: t}\right)$. Assuming that we want to evaluate $I(f)$ then, provided that the support of $q\left(x_{0: t} \mid y_{1: t}\right)$ includes the support of $p\left(x_{0: t} \mid y_{1: t}\right)$, we get the following identity

$$
I(f)=\frac{\int f\left(x_{0: t}\right) \widetilde{w}\left(x_{0: t}\right) q\left(x_{0: t} \mid y_{1: t}\right) d x_{0: t}}{\int \widetilde{w}\left(x_{0: t}\right) q\left(x_{0: t} \mid y_{1: t}\right) d x_{0: t}}
$$

where $w\left(x_{0: t}\right)$ is known as the importance weight

$$
\widetilde{w}\left(x_{0: t}\right)=\frac{p\left(x_{0: t} \mid y_{1: t}\right)}{q\left(x_{0: t} \mid y_{1: t}\right)}
$$

Consequently, if one can simulate $N$ i.i.d. particles $\left\{x_{0: t}^{(i)}, i=1, \ldots, N\right\}$ according to $q\left(x_{0: t} \mid y_{1: t}\right)$, a possible Monte Carlo estimate of $I(f)$ is

$$
\widehat{I}_{N}(f)=\frac{\frac{1}{N} \sum_{i=1}^{N} f\left(x_{0: t}^{(i)}\right) \widetilde{w}\left(x_{0: t}^{(i)}\right)}{\frac{1}{N} \sum_{j=1}^{N} \widetilde{w}\left(x_{0: t}^{(i)}\right)}=\sum_{i=1}^{N} f\left(x_{0: t}^{(i)}\right) w_{t}^{(i)}
$$

where the normalized importance weights $w_{t}^{(i)}$ are given by

$$
w_{t}^{(i)}=\frac{\widetilde{w}\left(x_{0: t}^{(i)}\right)}{\sum_{j=1}^{N} \widetilde{w}\left(x_{0: t}^{(j)}\right)}
$$

For $N$ finite, $\widehat{I}_{N}()$ is biased (ratio of two estimates) but asymptotically, under weak assumptions, the strong law of large numbers applies, that is $\widehat{I}_{N}(f) \underset{N \rightarrow+\infty}{\stackrel{a . s}{\rightarrow}} I(f)$. Under additional assumptions a central limit theorem, with a convergence rate independent of the dimension of the integrand, can be obtained [19]. It is clear that this integration method can also be interpreted as a sampling method where the posterior distribution $p\left(x_{0: t} \mid y_{1: t}\right)$ is approximated by

$$
\widehat{P}_{N}\left(d x_{0: t} \mid y_{1: t}\right)=\sum_{i=1}^{N} w_{t}^{(i)} \delta_{x_{0: t}^{(i)}}\left(d x_{0: t}\right)
$$

A recursive (sequential in time) procedure may be obtained by adopting the following proposal distribution:

$$
q\left(x_{0: t} \mid y_{1: t}\right)=q\left(x_{0: t-1} \mid y_{1: t-1}\right) q\left(x_{t} \mid x_{0: t-1}, y_{1: t}\right)
$$

and only proposing candidates $x_{t}$ at time $t$. That is the past is not changed. From equation (1) (ignoring $z$ ), it is easy to see that this importance function allows us to obtain the following recursive estimate of the importance weights at time $t$ :

$$
w_{t}^{(i)} \propto \frac{p\left(y_{t} \mid x_{t}^{(i)}\right) p\left(x_{t}^{(i)} \mid x_{0: t-1}^{(i)}, y_{1: t-1}\right)}{q\left(x_{t}^{(i)} \mid x_{0: t-1}^{(i)}, y_{1: t}\right)}
$$

In our JMLG model setting, we use a weighted set of samples (particles) $\left\{\left(x_{0: t}^{(i)}, z_{0: t}^{(i)}\right), w_{t}^{(i)}\right\}_{i=1}^{N}$ to approximate the posterior with the following point-mass distribution

$$
\widehat{P}_{N}\left(d x_{0: t}, z_{0: t} \mid y_{1: t}\right)=\sum_{i=1}^{N} w_{t}^{(i)} \delta_{x_{0: t}^{(i)}, z_{0: t}^{(i)}}\left(d x_{0: t}, z_{0: t}\right),
$$


where $\delta_{x_{0: t}^{(i)}, z_{0: t}^{(i)}}\left(d x_{0: t}, z_{0: t}\right)$ denotes the Dirac-delta function. Given $N$ particles $\left\{x_{0: t-1}^{(i)}, z_{0: t-1}^{(i)}\right\}_{i=1}^{N}$ at time $t-1$, approximately distributed according to $p\left(d x_{0: t-1}^{(i)}, z_{0: t-1}^{(i)} \mid y_{1: t-1}\right), \mathrm{PF}$ enables us to compute $N$ particles $\left\{x_{0: t}^{(i)}, z_{0: t}^{(i)}\right\}_{i=1}^{N}$ approximately distributed according to $P\left(d x_{0: t}^{(i)}, z_{0: t}^{(i)} \mid y_{1: t}\right)$, at time $t$. Since we cannot sample from the posterior directly, the PF update is accomplished by introducing an appropriate importance proposal distribution $q\left(d x_{0: t}, z_{0: t}\right)$ from which we can obtain samples. The basic algorithm, Figure 5 (see Figure 6 for a graphical representation), consists of two steps: sequential importance sampling and a selection that allows us to choose the fittest particles at each time step (see the introductory chapter in [7] for a more detailed explanation of particle filters). This algorithm uses the transition priors as proposal distributions; $q\left(x_{0: t}, z_{0: t} \mid y_{1: t}\right)=p\left(x_{t} \mid x_{t-1}, z_{t}\right) p\left(z_{t} \mid z_{0: t-1}, x_{0: t-1}, y_{1: t-1}\right)$. For the selection step, we used a state-of-the-art minimum variance resampling algorithm [20].

Sequential importance sampling step

- For $i=1, \ldots, N$, sample from the transition priors

$$
\begin{aligned}
& \widehat{z}_{t}^{(i)} \sim P\left(z_{t} \mid z_{0: t-1}^{(i)}, x_{0: t-1}^{(i)}, y_{1: t-1}\right) \\
& \widehat{x}_{t}^{(i)} \sim P\left(d x_{t} \mid x_{t-1}^{(i)}, z_{t}^{(i)}\right)
\end{aligned}
$$

and set $\left(\widehat{x}_{0: t}^{(i)}, \widehat{z}_{0: t}^{(i)}\right) \triangleq\left(\widehat{x}_{t}^{(i)}, \widehat{z}_{t}^{(i)}, x_{0: t-1}^{(i)}, z_{0: t-1}^{(i)}\right)$.

- For $i=1, \ldots, N$, evaluate and normalize the importance weights

$$
w_{t}^{(i)} \propto p\left(y_{t} \mid \widehat{x}_{t}^{(i)}, \widehat{z}_{t}^{(i)}\right)
$$

Selection step

- Multiply/Discard particles $\left\{\widehat{x}_{0: t}^{(i)}, \widehat{z}_{0: t}^{(i)}\right\}_{i=1}^{N}$ with respect to high/low importance weights $w_{t}^{(i)}$ to obtain $N$ particles $\left\{x_{0: t}^{(i)}, z_{0: t}^{(i)}\right\}_{i=1}^{N}$.

Fig. 5. PF algorithm at time $t$.

\section{RaO-BlackWellised Particle Filtering}

By considering the following factorization [9]:

$$
p\left(x_{0: t}, z_{0: t} \mid y_{1: t}\right)=p\left(x_{0: t} \mid y_{1: t}, z_{0: t}\right) p\left(z_{0: t} \mid y_{1: t}\right),
$$

it is possible to design algorithms with less variance. The density $p\left(x_{0: t} \mid y_{1: t}, z_{0: t}\right)$ is Gaussian (given $z_{t}$, the JMLG model reduces to a simple linear Gaussian model) and can be computed analytically if we know the marginal posterior density $p\left(z_{0: t} \mid y_{1: t}\right)$. This density satisfies the alternative recursion

$$
\begin{aligned}
& p\left(z_{0: t} \mid y_{1: t}\right)=p\left(z_{0: t-1} \mid y_{1: t-1}\right) \\
& \quad \times \frac{p\left(y_{t} \mid y_{1: t-1}, z_{0: t}\right) p\left(z_{t} \mid z_{0: t-1}, y_{1: t-1}\right)}{p\left(y_{t} \mid y_{1: t-1}\right)}
\end{aligned}
$$

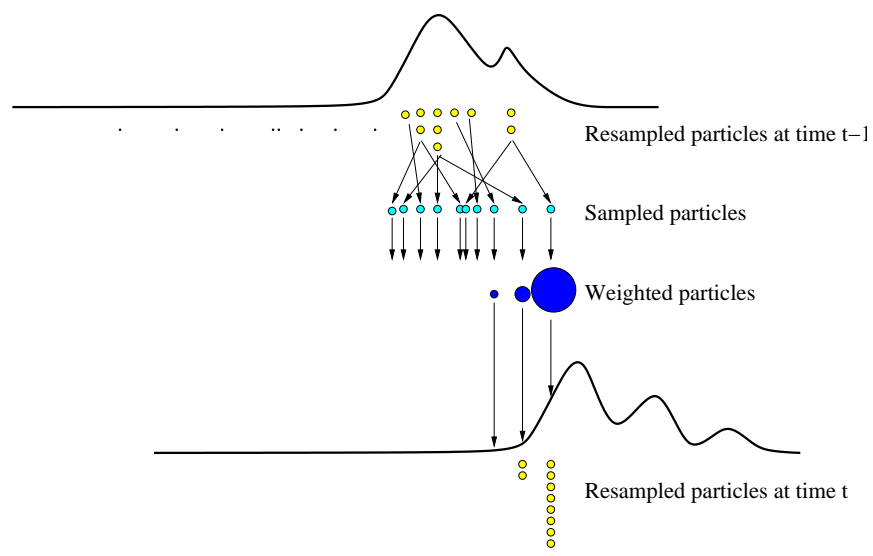

Fig. 6. Graphical representation of the PF algorithm for a continuous onedimensional problem. Starting with the resampled particles at $t-1$, a new set of particles is proposed at time $t$. We compute the importance weight of each particle. Finally, we select the fi ttest particles according to their weights. Note the fi lter has failed to track the two modes appearing on the right of the fi ltering posterior distribution at time $t$.

If equation (1) does not admit a closed-form expression, then equation (5) does not admit one either and samplingbased methods are still required. (Also note that the term $p\left(y_{t} \mid y_{1: t-1}, z_{0: t}\right)$ in equation (5) does not simplify to $p\left(y_{t} \mid z_{t}\right)$ because there is a dependency on past values through $x_{0: t}$.) This is immediately apparent if we look at the graphical model representation in Figure 3.

Now assuming that we can use a weighted set of samples $\left\{z_{0: t}^{(i)}, w_{t}^{(i)}\right\}_{i=1}^{N}$ to represent the marginal posterior distribution

$$
\widehat{P}_{N}\left(z_{0: t} \mid y_{1: t}\right)=\sum_{i=1}^{N} w_{t}^{(i)} \delta_{z_{0: t}^{(i)}}\left(z_{0: t}\right)
$$

the marginal density of $x_{0: t}$ is a Gaussian mixture

$$
\begin{aligned}
\widehat{p}_{N}\left(x_{0: t} \mid y_{1: t}\right) & =\int p\left(x_{0: t} \mid z_{0: t}, y_{1: t}\right) d P\left(z_{0: t} \mid y_{1: t}\right) \\
& =\int p\left(x_{0: t} \mid z_{0: t}, y_{1: t}\right) \sum_{i=1}^{N} w_{t}^{(i)} \delta_{z_{0: t}^{(i)}}\left(z_{0: t}\right) \\
& =\sum_{i=1}^{N} w_{t}^{(i)} p\left(x_{0: t} \mid y_{1: t}, z_{0: t}^{(i)}\right)
\end{aligned}
$$

that can be computed efficiently with a stochastic bank of Kalman filters. That is, we use PF to estimate the distribution of $z_{t}$ and exact computations (Kalman filters - one for each particle) to estimate the mean and variance of $x_{t}$. In particular, we sample $z_{t}^{(i)}$ and then propagate the mean $\mu_{t}^{(i)}$ and covariance $\Sigma_{t}^{(i)}$ of $x_{t}$ with a Kalman filter:

$$
\begin{aligned}
\mu_{t \mid t-1}^{(i)} & =A\left(z_{t}^{(i)}\right) \mu_{t-1}^{(i)}+F\left(z_{t}^{(i)}\right) u_{t} \\
\Sigma_{t \mid t-1}^{(i)} & =A\left(z_{t}^{(i)}\right) \Sigma_{t-1}^{(i)} A\left(z_{t}^{(i)}\right)^{\mathrm{T}}+B\left(z_{t}^{(i)}\right) B\left(z_{t}^{(i)}\right)^{\mathrm{T}} \\
S_{t}^{(i)} & =C\left(z_{t}^{(i)}\right) \Sigma_{t \mid t-1}^{(i)} C\left(z_{t}^{(i)}\right)^{\mathrm{T}}+D\left(z_{t}^{(i)}\right) D\left(z_{t}^{(i)}\right)^{\mathrm{T}} \\
y_{t \mid t-1}^{(i)} & =C\left(z_{t}^{(i)}\right) \mu_{t \mid t-1}^{(i)}+G\left(z_{t}^{(i)}\right) u_{t} \\
\mu_{t}^{(i)} & =\mu_{t \mid t-1}^{(i)}+\Sigma_{t \mid t-1}^{(i)} C\left(z_{t}^{(i)}\right)^{\mathrm{T}} S_{t}^{-1(i)}\left(y_{t}-y_{t \mid t-1}^{(i)}\right) \\
\Sigma_{t}^{(i)} & =\Sigma_{t \mid t-1}^{(i)}-\Sigma_{t \mid t-1}^{(i)} C\left(z_{t}^{(i)}\right)^{\mathrm{T}} S_{t}^{-1(i)} C\left(z_{t}^{(i)}\right) \Sigma_{t \mid t-1}^{(i)},
\end{aligned}
$$


where,

- $\mu_{t \mid t-1} \triangleq \mathbb{E}\left(x_{t} \mid y_{1: t-1}\right)$

- $\mu_{t} \triangleq \mathbb{E}\left(x_{t} \mid y_{1: t}\right)$

- $y_{t \mid t-1} \triangleq \mathbb{E}\left(y_{t} \mid y_{1: t-1}\right)$

- $\Sigma_{t \mid t-1} \triangleq \operatorname{cov}\left(x_{t} \mid y_{1: t-1}\right)$

- $\Sigma_{t} \triangleq \operatorname{cov}\left(x_{t} \mid y_{1: t}\right)$

- $S_{t} \triangleq \operatorname{cov}\left(y_{t} \mid y_{1: t-1}\right)$.

Hence, using the prior proposal for $z_{t}$ and applying equation (5), we find that the importance weights for $z_{t}$ are given by the predictive density

$$
p\left(y_{t} \mid y_{1: t-1}, z_{1: t}\right)=\mathcal{N}\left(y_{t} ; y_{t \mid t-1}, S_{t}\right) .
$$

This RBPF algorithm was applied to fault diagnosis in [21]. The algorithm is shown in Figure 7.

Sequential importance sampling step

- For $i=1, \ldots, N$, set $\widehat{\mu}_{t \mid t-1}^{(i)} \triangleq \mu_{t \mid t-1}^{(i)}, \widehat{\Sigma}_{t \mid t-1}^{(i)} \triangleq \Sigma_{t \mid t-1}^{(i)}$, sample

$$
\widehat{z}_{t}^{(i)} \sim \operatorname{Pr}\left(z_{t} \mid x_{0: t-1}^{(i)}, z_{0: t-1}^{(i)}, y_{1: t-1}\right)
$$

- For $i=1, \ldots, N$, evaluate and normalize the importance weights

$$
w_{t}^{(i)} \propto p\left(y_{t} \mid y_{1: t-1}, \widehat{z}_{t}^{(i)}\right)
$$

$\underline{\text { Selection step }}$

- Multiply/Discard particles $\left\{\widehat{\mu}_{t \mid t-1}^{(i)}, \widehat{\Sigma}_{t \mid t-1}^{(i)}, \widehat{z}_{t}^{(i)}\right\}_{i=1}^{N}$ with respect to high/low importance weights $w_{t}^{(i)}$ to obtain $N$ particles $\left\{\mu_{t \mid t-1}^{(i)}, \Sigma_{t \mid t-1}^{(i)}, z_{t}^{(i)}\right\}_{i=1}^{N}$

\section{Updating step}

- For $i=1, \ldots, N$, use one step of the Kalman recursion to compute the sufficient statistics $\quad\left\{\mu_{t+1 \mid t}^{(i)}, \Sigma_{t+1 \mid t}^{(i)}, y_{t+1 \mid t}^{(i)}, S_{t+1}^{(i)},\right\} \quad$ given $\left\{z_{t}^{(i)}, \mu_{t \mid t-1}^{(i)}, \Sigma_{t \mid t-1}^{(i)}\right\}$.

Fig. 7. RBPF algorithm at time $t$. For each particle $z_{t}^{(i)}$ we propagate the mean and variance of $x_{t}$. This enables us to approximate the posterior of $x$ with a mixture of Gaussians. Note that in the simple PF, the posterior is approximated with a more coarse model: a mixture of Dirac-delta functions.

The RBPF requires that we propagate a Kalman filter for each particle. It appears at first sight to be computational expensive. The experiments will show, however, that this extra computation per particle can result in vast improvements in accuracy and robustness.

\section{LOOK-AhEAD RAO-BLACKWELlised PARTICLE FILTERING}

In order to deal with unexpected observations, it is possible to improve the RBPF by looking one step ahead. One can expand the expression for the importance weights as follows:

$$
\begin{aligned}
w_{t} & =\frac{p\left(z_{0: t} \mid y_{1: t}\right)}{q\left(z_{0: t} \mid y_{1: t}\right)} \\
& =\frac{p\left(z_{0: t-1} \mid y_{1: t}\right)}{p\left(z_{0: t-1} \mid y_{1: t-1}\right)} \frac{p\left(z_{t} \mid z_{0: t-1}, y_{1: t}\right)}{q\left(z_{t} \mid z_{0: t-1}, y_{1: t}\right)} \\
& \propto \frac{p\left(y_{t} \mid y_{1: t-1}, z_{0: t}\right) p\left(z_{t} \mid z_{0: t-1}, y_{1: t-1}\right)}{q\left(z_{t} \mid z_{0: t-1}, y_{1: t}\right)} .
\end{aligned}
$$

The choice of proposal distribution, $q\left(z_{0: t} \mid y_{1: t}\right)=$ $q\left(z_{t} \mid z_{0: t-1}, y_{1: t}\right) p\left(z_{0: t-1} \mid y_{1: t-1}\right)$, states that we are not sampling past trajectories. Sampling past trajectories requires solving an intractable integral [22].

We could use the transition prior as proposal distribution: $q\left(z_{t} \mid z_{0: t-1}, y_{1: t}\right)=p\left(z_{t} \mid z_{0: t-1}, y_{1: t-1}\right)$. Then, according to equation (10), the importance weights simplify to the predictive density of equation (7).

However, according to equation (9), the optimal proposal distribution corresponds to the choice $q\left(z_{t} \mid z_{0: t-1}, y_{1: t}\right)=$ $p\left(z_{t} \mid z_{0: t-1}, y_{1: t}\right)$. This distribution satisfies Bayes rule:

$$
p\left(z_{t} \mid z_{0: t-1}, y_{1: t}\right)=\frac{p\left(y_{t} \mid y_{1: t-1}, z_{0: t}\right) p\left(z_{t} \mid z_{0: t-1}, y_{1: t-1}\right)}{p\left(y_{t} \mid y_{1: t-1}, z_{0: t-1}\right)}
$$

and, hence, the importance weights simplify to

$$
\begin{aligned}
w_{t} & \propto p\left(y_{t} \mid y_{1: t-1}, z_{0: t-1}\right) \\
& \propto \sum_{z_{t}=1}^{n_{z}} p\left(y_{t} \mid y_{1: t-1}, z_{0: t-1}, z_{t}\right) p\left(z_{t} \mid z_{0: t-1}, y_{1: t-1}\right)
\end{aligned}
$$

When the number of discrete states is small, say 10 or 1000, we can compute the distributions in equations (11) and (12) analytically. That is, if the number of discrete states is reasonably small, we can compute the sums by simple enumeration. In addition to Rao-Blackwellization, this leads to substantial improvements over standard particle filters. Yet, a further improvement can still be attained.

Even when using the optimal importance distribution, there is a discrepancy arising from the ratio $p\left(z_{0: t-1} \mid y_{1: t}\right) / p\left(z_{0: t-1} \mid y_{1: t-1}\right)$ in equation (9). This discrepancy is what causes the well known problem of sample impoverishment in all particle filters [7], [23]. To circumvent it to a significant extent, one can note that the importance weights do not depend on $z_{t}$ (we are marginalising over this variable). It is therefore possible to select particles before the sampling step. That is, one chooses the fittest particles at time $t-1$ using the information at time $t$. This leads to an efficient algorithm (look-ahead RBPF), [15], [2] , whose pseudocode is shown in Figure 8. Note that for standard $\mathrm{PF}$, Figure 5, the importance weights depend on the sample $z_{t}^{(i)}$, thus not permitting selection before sampling. Selecting particles before sampling results in a richer sample set at the end of each time step. See Figure 9 for a graphical representation. 
Kalman prediction step

- For $\mathrm{i}=1, \ldots, \mathrm{N}$, and for $z_{t}=1, \ldots, n_{z}$ compute

$$
\widehat{\mu}_{t \mid t-1}^{(i)}\left(z_{t}\right), \quad \widehat{\Sigma}_{t \mid t-1}^{(i)}\left(z_{t}\right), \quad \widehat{y}_{t \mid t-1}^{(i)}\left(z_{t}\right), \quad \widehat{S}_{t}^{(i)}\left(z_{t}\right)
$$

- For $\mathrm{i}=1, \ldots, \mathrm{N}$, evaluate and normalize the importance weights

$$
\begin{aligned}
w_{t}^{(i)} & =p\left(y_{t} \mid y_{1: t-1}, z_{0: t-1}^{(i)}\right) \\
& =\sum_{z_{t}=1}^{n_{z}} \mathcal{N}\left(\widehat{y}_{t \mid t-1}^{(i)}\left(z_{t}\right), \widehat{S}_{t}^{(i)}\left(z_{t}\right)\right) p\left(z_{t} \mid z_{0: t-1}^{(i)}, y_{1: t-1}\right)
\end{aligned}
$$

Selection step

- Multiply/Discard particles $\left\{\widehat{\mu}_{t-1}^{(i)}, \widehat{\Sigma}_{t-1}^{(i)}, \widehat{z}_{0: t-1}^{(i)}\right\}_{i=1}^{N}$ with respect to high/low importance weights $w_{t}^{(i)}$ to obtain $N$ particles $\left\{\mu_{t-1}^{(i)}, \Sigma_{t-1}^{(i)}, z_{0: t-1}^{(i)}\right\}_{i=1}^{N}$

Sequential importance sampling step

- Kalman prediction. For $\mathrm{i}=1, \ldots, \mathrm{N}$, and for $z_{t}=1, \ldots, n_{z}$ using the resampled information, re-compute

$$
\widehat{\mu}_{t \mid t-1}^{(i)}\left(z_{t}\right), \quad \widehat{\Sigma}_{t \mid t-1}^{(i)}\left(z_{t}\right), \quad \widehat{y}_{t \mid t-1}^{(i)}\left(z_{t}\right), \quad \widehat{S}_{t}^{(i)}\left(z_{t}\right)
$$

- For $z_{t}=1, \ldots, n_{z}$ compute

$$
p\left(z_{t} \mid z_{0: t-1}^{(i)}, y_{1: t}\right) \propto \mathcal{N}\left(\widehat{y}_{t \mid t-1}^{(i)}\left(z_{t}\right), \widehat{S}_{t}^{(i)}\left(z_{t}\right)\right) p\left(z_{t} \mid z_{0: t-1}^{(i)}, y_{1: t-1}\right)
$$

- Sampling step

$$
z_{t}^{(i)} \sim p\left(z_{t} \mid z_{0: t-1}^{(i)}, y_{1: t}\right)
$$

Updating step

- For $\mathrm{i}=1, \ldots, \mathrm{N}$, use one step of the Kalman recursion to compute the sufficient statistics $\left\{\mu_{t}^{(i)}, \Sigma_{t}^{(i)}\right\}$ given $\left\{\widehat{\mu}_{t \mid t-1}\left(z_{t}^{(i)}\right), \widehat{\Sigma}_{t \mid t-1}\left(z_{t}^{(i)}\right)\right\}$.

Fig. 8. Look-ahead RBPF algorithm at time $t$. The algorithm uses an optimal proposal distribution. It also selects particles from time $t-1$ using the information at time $t$.

\section{EXPERIMENTS WITH B-14 RESEARCH ROBOT}

Jos'e is a general purpose research robot, specifically a Real World Interfaces B-14 mobile robot with a B-12 base, who recently won the AAAI Hors d'Oeuvres Competition [5] (see Figure 1).

The base unit contains two separate motors, one for translation and one for rotation. Power is provided by a rechargeable battery. The translational motor drives all three wheels, giving excellent traction. The rotational motor turns all three wheels in unison, so they are always pointing the same way. Thus there is no concept of "front" or "back" wheels - a given wheel can end up in the front (relative to the direction of travel), at the back, or anywhere in between. Jos'e can move along curved paths by simultaneously translating and rotating.

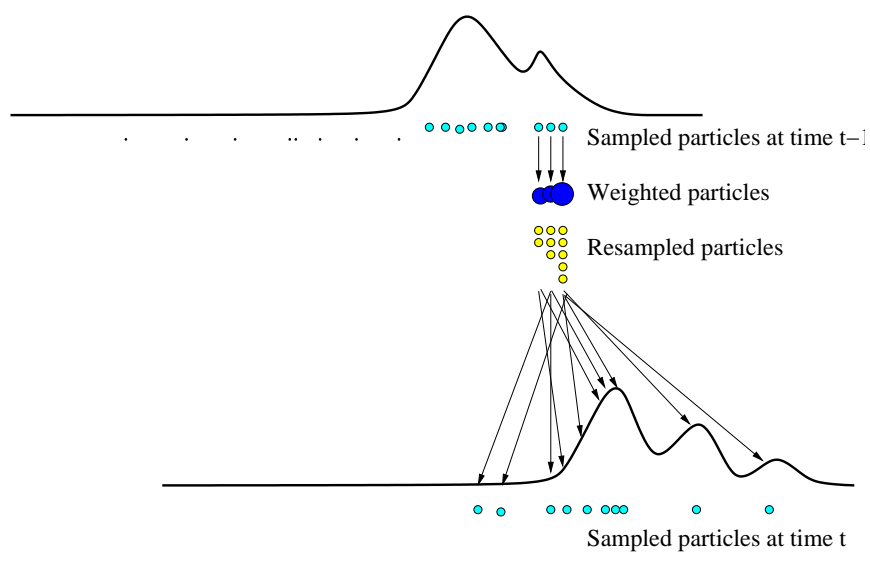

Fig. 9. Graphical representation of look-ahead RBPF. First we compute the importance weights. After resampling according to these weights, we propose new particles at time $t$. With this algorithm, we are more likely to be able to track all the modes of the changing filtering distribution.

The B-12's motors are stepping motors, controlled by a varying pulse width. Actual translation and rotational speed are measured by optical shaft encoders - one for translation and one for rotation. Individual wheels do not have separate encoders, so are assumed to all be translating or rotating at the same speed. There are commands to set the desired speed and acceleration. A feedback control loop monitors the actual values and adjusts the motor pulse width to achieve the desired values. Simpler open-loop commands are also available - these bypass the control loop and send a fixed pulse width directly to the motors.

The B-12 provides status reports including measured translational and rotational speeds, current and pulse width to either motor, battery voltage, etc. Jos'e also has a stereo camera and bump, infrared, and sonar sensors.

\section{A. Experimental Setup}

Using the currents as control signals $u_{t}$, we measured the speeds of the wheels $y_{t}$. We used a one dimensional continuous state $x_{t}$. This state need not have a physical interpretation (this is indeed not needed in our fault diagnosis setting). It should rather be interpreted as a projection of $y_{t}$ to a noiseless space. We performed tests using two sets of four discrete states $z_{t}$, chosen as follows:

\begin{tabular}{|c|l|}
\hline State & Description \\
\hline 1 & Smooth floor, no extra load \\
2 & Smooth floor, dragging a light load \\
3 & Smooth floor, dragging a medium load \\
4 & Smooth floor, dragging a heavy load \\
5 & Tiled floor, no extra load \\
6 & Tiled floor, dragging a light load \\
7 & Tiled floor, dragging a medium load \\
8 & Tiled floor, dragging a heavy load \\
\hline
\end{tabular}

The 'dragging' states were chosen so that we could repeatably simulate a fault situation in which Jos'e has bumped into 
or snagged something. The smooth floor represents a lownoise environment, while the tiled floor represents a noisy environment.

We engineered the initial and transition matrices for the discrete states $z_{0} \sim p\left(z_{0}\right)$ and $p\left(z_{t} \mid z_{t-1}\right)$, collected data in each discrete state and learned the matrices $A, C, F, G$ using a conventional optimization process and a standard procedure in control engineering [24]. The noise matrices $B$ and $D$ were learned via the expectation maximisation (EM) algorithm for linear dynamic models [16]. Figure 10 shows the measured data and data generated by the JMLG model under several step responses.
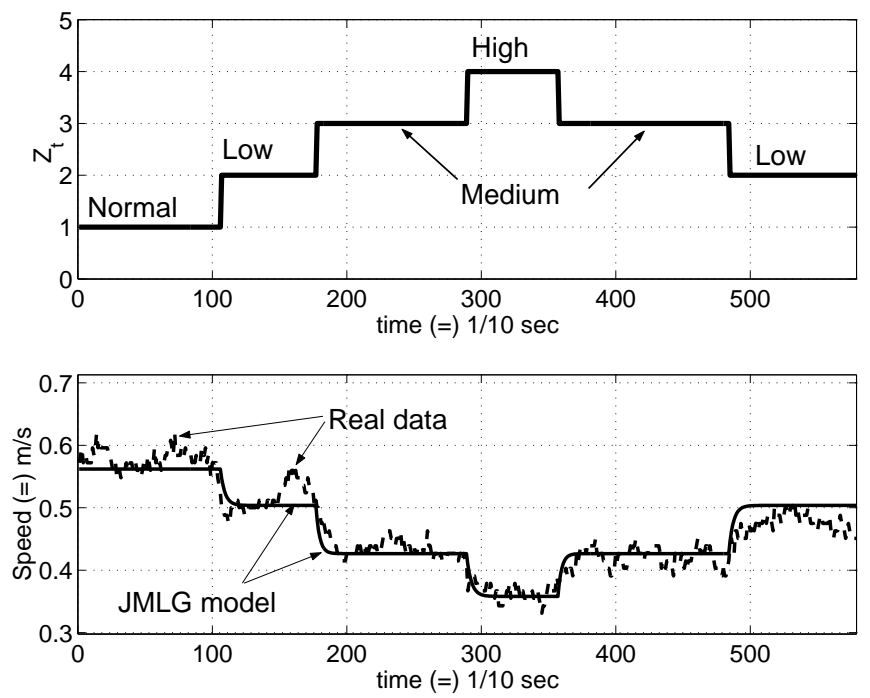

Fig. 10. To test how well our models capture the behaviour of the data, we used them to generate data under a switching load $z_{t}$. As shown in the plot below, the generated data $y_{t}$ captures the trends in the real data.

\section{B. Testing and Results}

Over 30 test runs were conducted, in which the robot was placed in different states at random times. Measurements were recorded every $0.1 \mathrm{sec}$. The three particle filtering algorithms were tested. For each dataset and algorithm, we plotted diagnosis error rate versus number of particles and CPU time. The diagnosis error rate is the percentage of time steps in which the diagnosed state differed from the actual state. This obviously decreases with the number of particles in all algorithms. Note that there is a baseline error rate resulting from human inaccuracy in timing the state changes.

1) Low-Noise Environment: Test runs involving state changes while the robot was travelling on the smooth floor produced the results shown in figures 12 and 11 . In this environment, la-RBPF is clearly superior. One would expect it to perform better than the other algorithms for the same number of particles; la-RBPF is doing a lot more work per particle. But even when the extra time per particle is factored in, la-RBPF is still more efficient. Overall it produces a lower

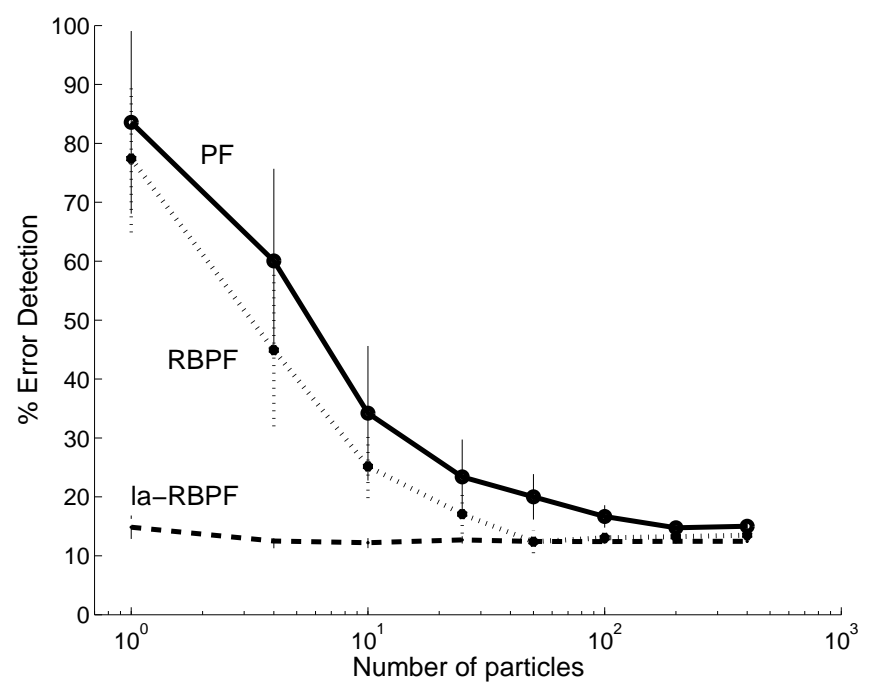

Fig. 11. Smooth fbor. Diagnosis error vs number of particles. Estimation based on 25 runs.

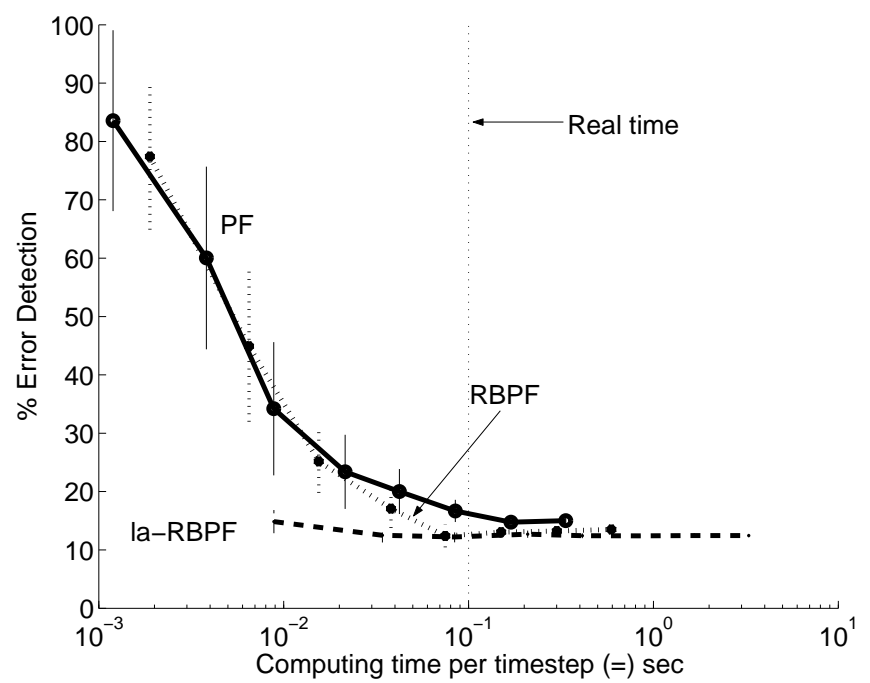

Fig. 12. Smooth fbor. Diagnosis error vs computing time. Estimation based on 25 runs.

error rate (and lower variance) than PF and RBPF using the same CPU time.

2) High-Noise Environment: Similar state changes while operating on a tiled floor yielded different results (see Figure 13). Here, la-RBPF's extra work resulted in no benefit. When we applied a simple moving-average filter to the data, la-RBPF's advantage was restored - Figure 14.

\section{EXPERIMENTS WITH MARS ROVERS}

\section{A. $K-9$ rover}

We performed experiments on a simple model of the suspension system of the K-9 rover at NASA Ames Research Center. $\mathrm{K}-9$, shown in Figure 15, is a six wheeled rover with a rockerbogey suspension, and we model the suspension's response to 


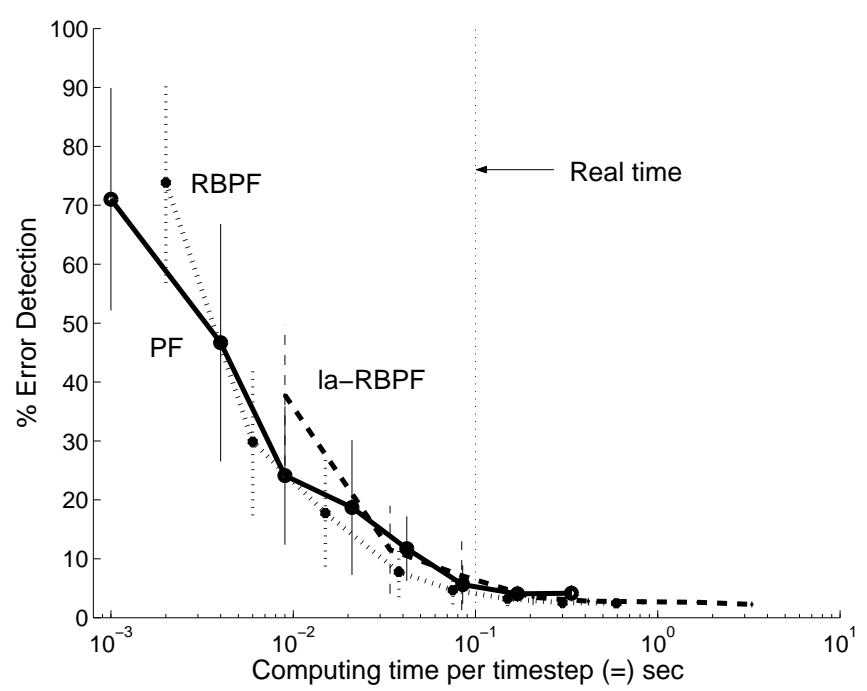

Fig. 13. Tiled fbor. Diagnosis error vs computing time. Estimation based on 25 runs.

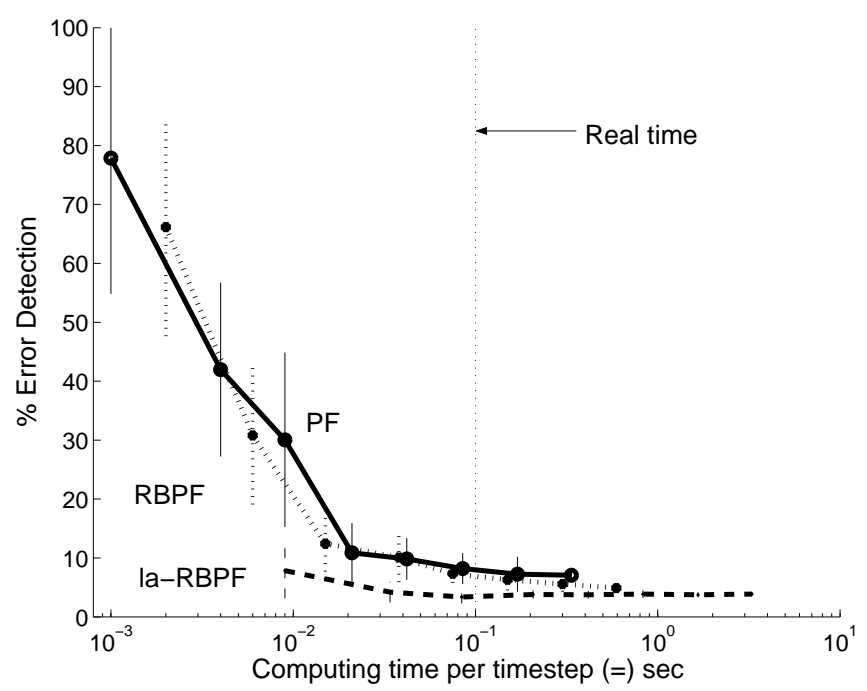

Fig. 14. Tiled fbor. Diagnosis error vs computing time, where the measurement signals have been filtered (smoothed). Estimation based on 25 runs.

driving over rocks and other obstacles to anticipate situations where the rover's scientific instruments could collide with an obstacle, or where the rover could become "high-centered" on a rock. The model has six discrete modes that refer to flat driving, rock under front wheel, rock under middle wheel, rock under rear wheel, rock between front and middle wheel and rock between middle and rear wheel, respectively. There are four continuous parameters in the model, which represent the rocker and bogey angles and their derivatives. The angles are the two observable variables of the system.

The trajectories of rocker and bogey angles $\alpha$ and $\beta$ can be modelled as trigonometric functions when one wheel is going over the rock and constant otherwise. By also modelling their derivatives $\dot{\alpha}$ and $\dot{\beta}$ as continuous parameters of the system, we obtain linear probabilistic transition functions of the form

$$
\alpha_{t}=\alpha_{t-1}+k \dot{\alpha}_{t-1}+N\left(0, \sigma_{\alpha}^{2}\right)
$$

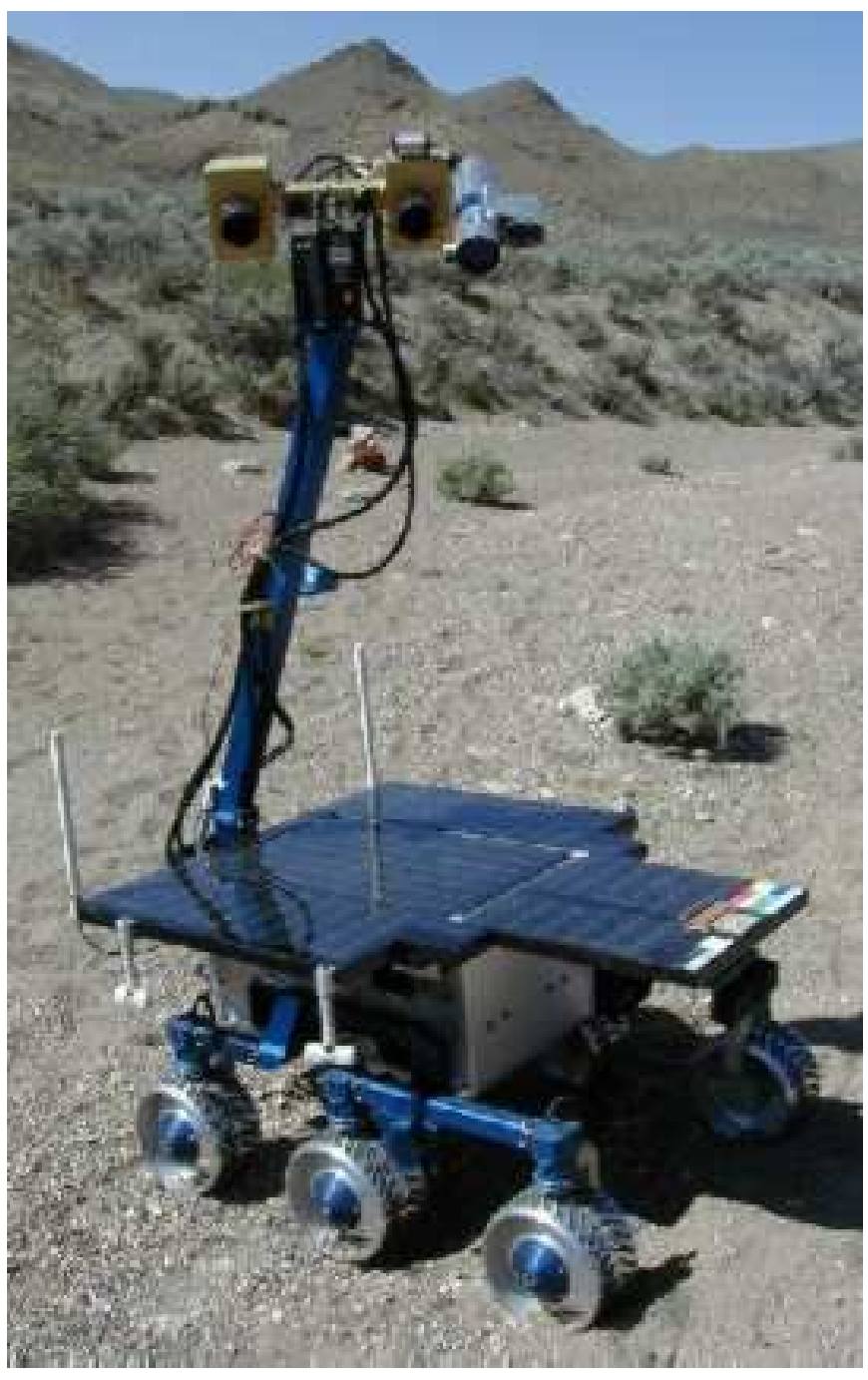

Fig. 15. The planetary rover K-9.

and

$$
\dot{\alpha_{t}}=\alpha_{t-1}+k \alpha_{t-1}+N\left(0, \sigma_{\dot{\alpha}}^{2}\right)
$$

and likewise for $\beta$. With $\ddot{\alpha}_{t}=-\alpha_{t}$ in the trigonometric case and $\ddot{\alpha}_{t}=0$ in the constant case all transition functions are linear functions of the system's continuous parameters. The discrete transition function and the noise terms were engineered by hand.

We carried out two sets of tests. In the first set, the model was used to generate data for which ground truth was available as to the true values of the mode and continuous variables, and the algorithms were applied to this artificial data. In the second setting, we applied the model to detect rocks in real data. This data was generated at NASA Ames by driving the K-9 rover over three different rocks in an outdoor "sandbox" consisting of gravel, sand, rocks and small hills.

We first report the results on artificial data with ground truth. Here, the look-ahead variant of RBPF proved to be highly efficient. In this set of tests, the diagnosis of every filter is taken to be the maximum a posteriori (MAP) estimate of the discrete modes. 


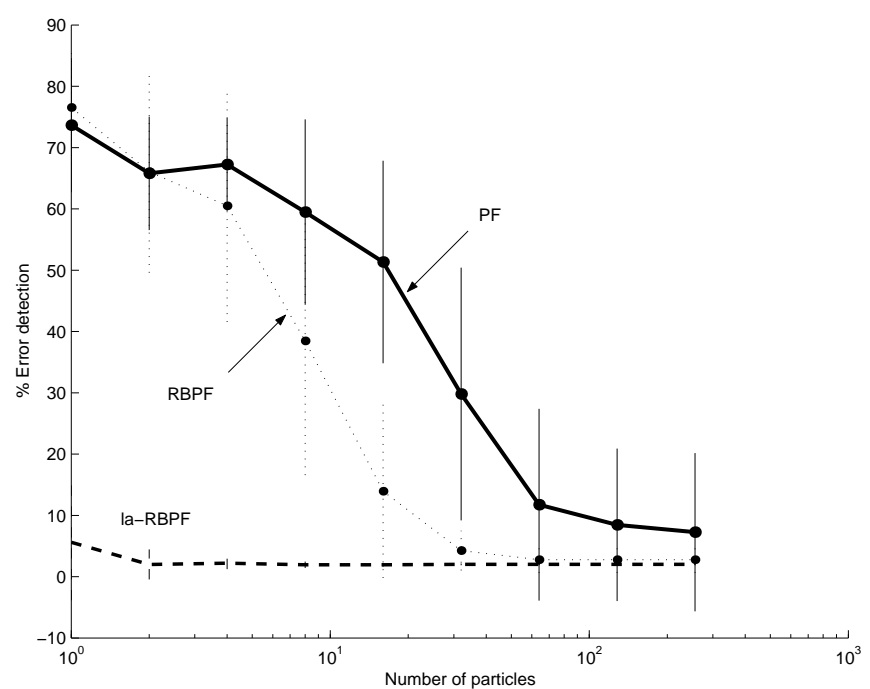

Fig. 16. Performance of PF, RBPF and la-RBPF. Estimation based on 50 runs.

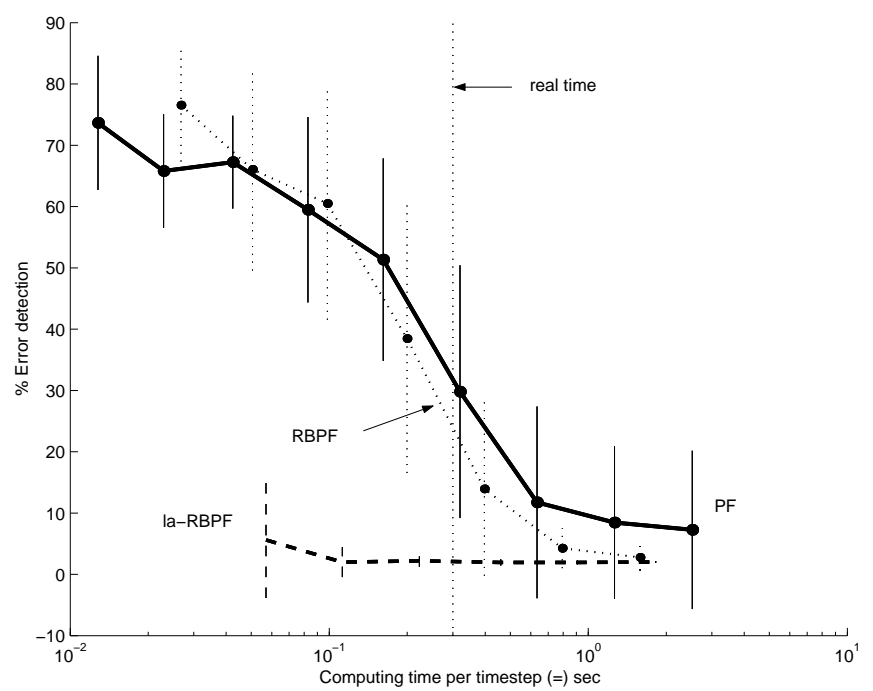

Fig. 17. Performance of PF, RBPF, and la-RBPF. Estimation based on 50 runs.

Figure 16 shows the percentage of incorrect diagnoses for PF, RBPF, and la-RBPF for a varying number of particles. Clearly, la-RBPF and RBPF yield better results than PF with the same number of particles. When we take into account the additional computation time la-RBPF and RBPF need per particle, the performance of PF and RBPF is comparable but the two are still clearly dominated by la-RBPF (see Figure 17).

In some cases we are not only interested in diagnosing the discrete modes, but also the continuous parameters of a hybrid system. As an example we might want to know the steepness of a hill, or the size of a rock we are driving over. In other applications, these continuous parameters might be crucial, particularly when diagnosing sensor defects and calibration problems.

Figure 18 shows the mean squared error (MSE) of the PF, RBPF and la-RBPF algorithms on the same artificial data as above where ground truth is available. The Y-scale is

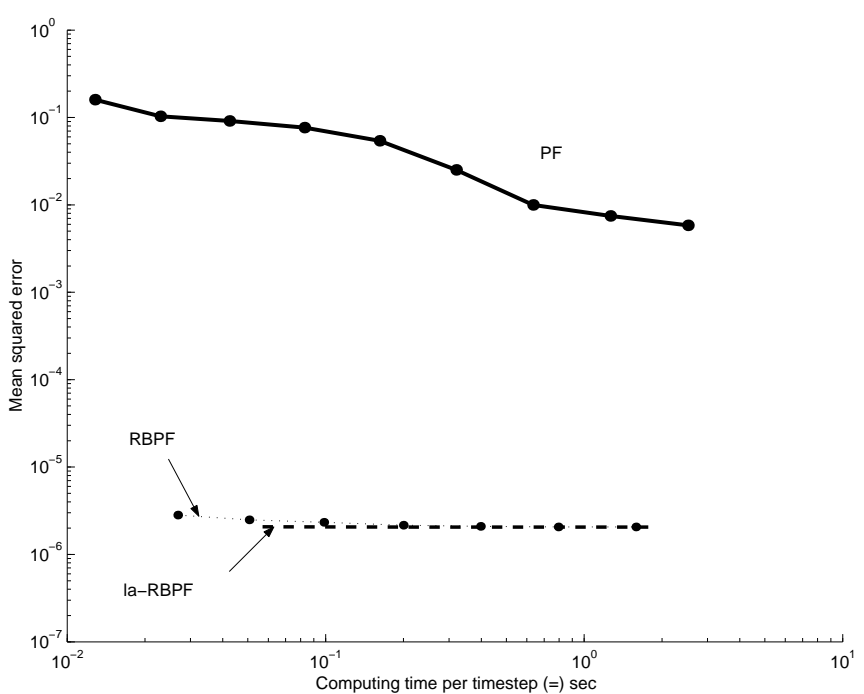

Fig. 18. Mean squared errors of the PF, RBPF and la-RBPF algorithms, averaged over 50 runs. Note the logarithmic scale for both the X- and Yaxes.

logarithmic to fit the extreme differences between PF and the other algorithms. With a mean squares estimation error of the continuous parameters which is between $10^{3}$ and $10^{5}$ times higher than that of la-RBPF, it is clearly outperformed. The difference between RBPF and la-RBPF is very small with the look-ahead variant performing slightly better for small numbers of particles.

We also applied the algorithms to real data from the K9 rover. In Figure 19, we show the two observed variables, rocker and bogey angle as well as the discrete mode estimates $\mathrm{PF}$ and la-RBPF yield with the number of particles they can roughly process in real time. PF uses 32 particles while laRBPF is allowed to use 4 particles. To avoid a cluttered picture we omit RBPF (with eight particles) which yields better results than PF but still performs worse than la-RBPF. To iterate, state 1 represents flat driving, state 2 driving over a rock with the front wheel, state 3 with the middle wheel and state 4 with the rear wheel. State 5 represents the rock being between the front and the middle wheel and state 6 between the middle and the rear wheel. There is no ground truth for this data, although it is fairly clear from the data when the rover drives over the three rocks. The PF almost misses the third rock and gets confused by little changes in the data during flat driving. The la-RBPF algorithm detects all the rocks and only the rocks, but on the second rock, the traversal of the rear wheel is missed. We believe this is due to weaknesses in the model rather than in the tracking algorithm.

\section{B. Marsokhod rover}

At NASA Ames Research Center, we also applied RaoBlackwellised Particle Filtering for model-based fault detection on the Marsokhod rover, a medium-sized planetary rover with six wheels (see Figure 2). The wheel model was initially only designed to demonstrate feasibility of model based diagnosis [25]. It is completely hand-crafted and the effort of tuning parameters was not undertaken. Despite this 


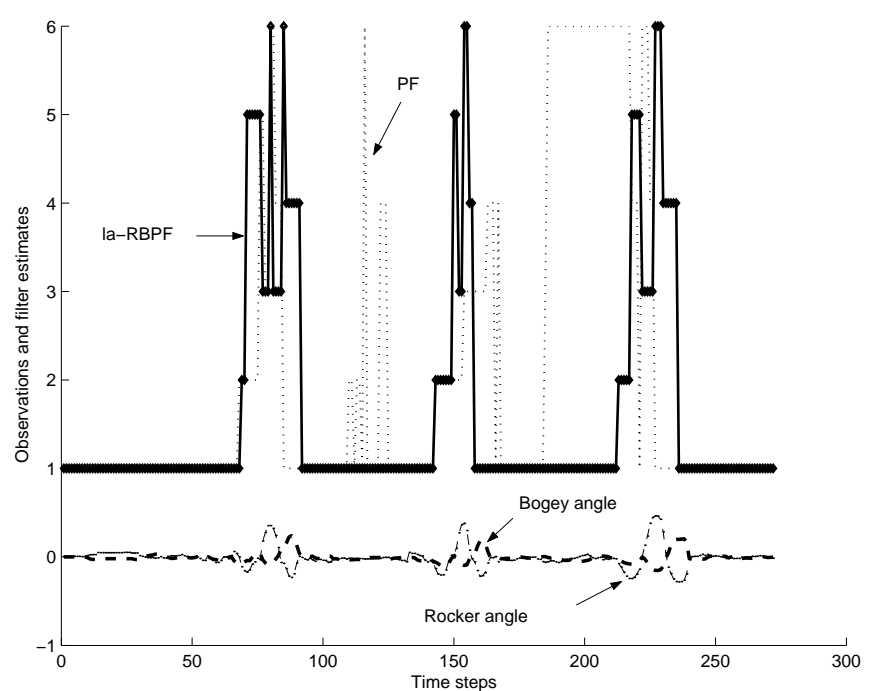

Fig. 19. Discrete mode estimates on real data.

fact, we can diagnose faults very efficiently and correctly using this model and Rao-Blackwellised particle filtering.

Diagnosis is run independently on each of the six wheels. There are 22 discrete states, 9 of which are normal operational states and 13 of which represent fault states. Diagnosable faults include a stalled motor, a broken gear, and a broken gear and encoder. For the experiments we describe here, the right rear wheel had a broken gear and encoder. The task of diagnosis was to reliably identify this fault with as little measurements as possible. The rover was set to an idle state in which the fault is not diagnosable and then it was given the command to start. We report the number of measurements the algorithms needed to settle for the diagnosis of a broken gear and encoder at the rear back wheel.

In Figure 20, we report this number of measurements on the $\mathrm{Y}$-axis as a function of the varying number of particles the algorithms are given (the total number of measurements after the command was issued was 46 and if the fault is never diagnosed we report this number). Standard Particle Filters show very inferior performance in this task. Only with 1000 particles and above they are able to diagnose the fault in some of the experiments. The look-ahead variant of RBPF, on the other hand, can already diagnose the fault with one single particle. It is simply tracking the mode very well. The picture does not change much when we take into account the different time complexities of the algorithms: la-RPBF is still performing considerably better than RBPF which in turn outperforms PF significantly. For additional information on the wheel model and more experiments we refer the reader to [25].

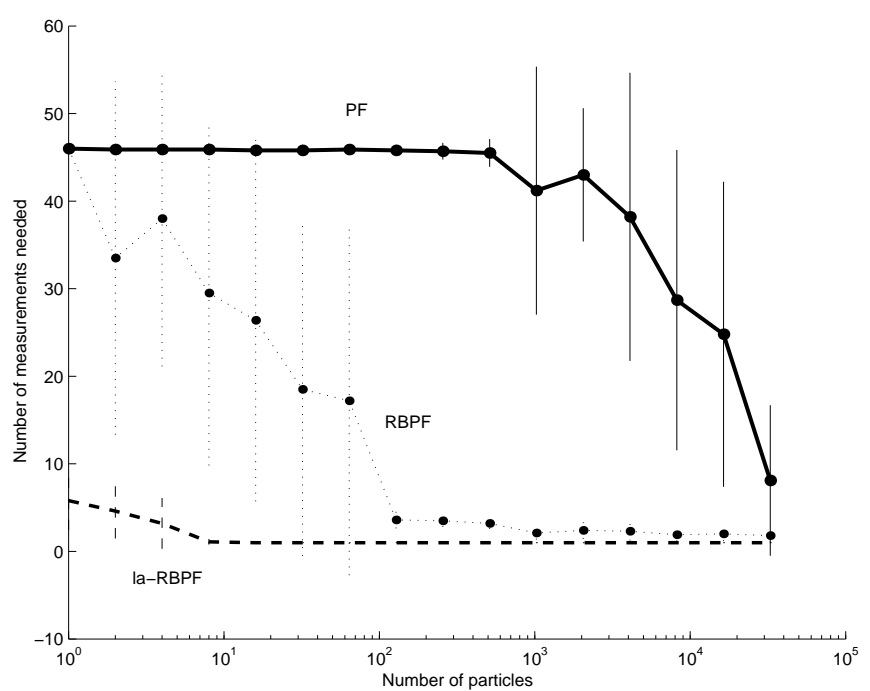

Fig. 20. Number of measurements needed to diagnose a fault. Algorithms PF, RBPF and la-RBPF with a varying number of particles available for diagnosis. Estimation based on 25 runs.

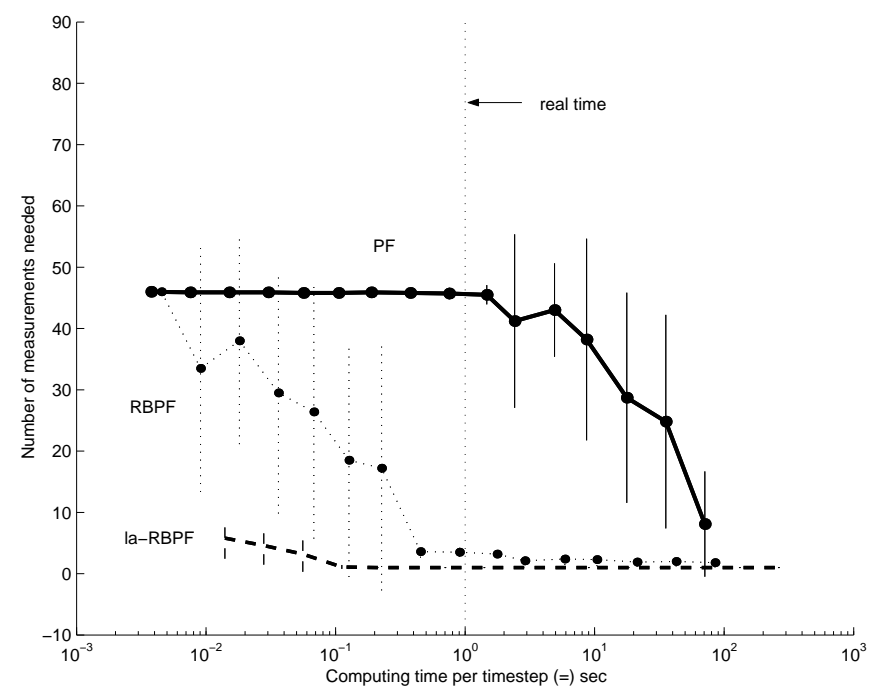

Fig. 21. Number of measurements needed to diagnose a fault. Algorithms PF, RBPF and la-RBPF with varying computation time given per time step. Estimation based on 25 runs.

\section{CONCLUSION}

In this paper, we demonstrated the use of algorithms, that combine particle and Kalman filters, for diagnosing mobile robots. In particular, we showed that the look-ahead RBPF can compute very accurate diagnosis probabilities in real-time. There are two reasons for this. First, marginalising continuous variables reduces the problem of high-dimensional state spaces considerably. Second, the look ahead feature is important in diagnosis with particle filters, where the probability of faulty states is typically low. Looking one step-ahead improves the probability of proposing particles in these states of low probability.

This state estimation method can also be used to select control strategies [26]. This is an essential step toward having robots that can carry out self-maintenance. 
There are several avenues for further research. Standard particle filters allow us to adopt nonlinear measurement models, but this comes with a great computational cost. Many current efforts are being devoted to the design of efficient filters to handle this situation [27], [28], [29]. We also need to investigate hierarchical models for specifying faults. The aim is to end up with sparse models that can be solved efficiently using multiple Rao-Blackwellisation. Finally, it is important to continue testing the robots, models and algorithms in more demanding conditions.

\section{ACKNOWLEDGMENT}

We thank the Jos'e team at UBC and, in particular, Pantelis Elinas and Don Murray for getting us started with Jos'e. We would also like to thank Arnaud Doucet for suggestions and Zoubin Ghahramani for his EM code for linear dynamic models. Nando de Freitas was supported by NSERC and IRISROPAR, Frank Hutter was partially supported by a RIACS SSRP student fellowship at NASA Ames Research Center, Ruben Morales-Men'endez was partially supported by the Government of Canada (ICCS) and UBC's CS department.

\section{REFERENCES}

[1] S. Thrun, J. Langford, and V. Verma, "Risk sensitive particle filters," in Advances in Neural Information Processing Systems 14, S. B. T. G Dietterich and Z. Ghahramani, Eds. Cambridge, MA: MIT Press, 2002.

[2] R. Morales-Menendez, N. de Freitas, and D. Poole, 'Real-time monitoring of complex industrial processes with particle fi lters," in Advances in Neural Information Processing Systems 16. Cambridge, MA: MIT Press, 2003.

[3] R. Dearden and D. Clancy, "Particle fi lters for real-time fault detection in planetary rovers," in International Workshop on Diagnosis, 2001.

[4] V. Verma, J. Fernandez, and R. Simmons, "Probabilistic models for monitoring and fault diagnosis," in The Second IARP and IEEE/RAS Joint workshop on technical challenges for dependable robots in human environment, R. Chatila, Ed., Toulouse, France, October 2002.

[5] P. Elinas, J. Hoey, D. Lahey, J. D. Montgomery, D. Murray, S. Se, and J. J. Little, "Waiting with Jos'e, a vision-based mobile robot." in International Conference on Robotics and Automation, 2002.

[6] N. Metropolis and S. Ulam, "The Monte Carlo method," Journal of the American Statistical Association, vol. 44, no. 247, pp. 335-341, 1949.

[7] A. Doucet, N. de Freitas, and N. J. Gordon, Eds., Sequential Monte Carlo Methods in Practice. Springer-Verlag, 2001.

[8] A. Doucet, N. de Freitas, K. Murphy, and S. Russell, "Rao-Blackwellised particle fi ltering for dynamic Bayesian networks," in Uncertainty in Artificial Intelligence, C. Boutilier and M. Godszmidt, Eds. Morgan Kaufmann Publishers, 2000, pp. 176-183.

[9] G. Casella and C. P. Robert, 'Rao-Blackwellisation of sampling schemes," Biometrika, vol. 83, no. 1, pp. 81-94, 1996.

[10] H. Akashi and H. Kumamoto, 'Random sampling approach to state estimation in switching environments," Automatica, vol. 13, pp. 429434, 1977.

[11] A. Doucet, 'On sequential simulation-based methods for Bayesian fi ltering," Department of Engineering, Cambridge University, Tech. Rep. CUED/F-INFENG/TR 310, 1998

[12] A. Doucet, S. Godsill, and C. Andrieu, 'On sequential Monte Carlo sampling methods for Bayesian fi ltering," Statistics and Computing, vol. 10, no. 3, pp. 197-208, 2000.

[13] A. Kong, J. S. Liu, and W. H. Wong, 'Sequential imputations and Bayesian missing data problems," Journal of the American Statistical Association, vol. 89, no. 425, pp. 278-288, 1994.

[14] S. N. MacEachern, M. Clyde, and J. S. Liu, 'Sequential importance sampling for nonparametric Bayes models: the next generation," Canadian Journal of Statistics, vol. 27, pp. 251-267, 1999.

[15] A. Doucet, N. J. Gordon, and V. Krishnamurthy, 'Particle fi lters for state estimation of jump Markov linear systems," Cambridge University Engineering Department, Tech. Rep. CUED/F-INFENG/TR 359, Sept. 1999.
[16] S. Roweis and Z. Ghahramani, "A unifying review of linear Gaussian models," Neural Computation, vol. 11, no. 2, pp. 305-345, 1999.

[17] R. H. Shumway and D. S. Stoffer, "An approach to time series smoothing and forecasting using the EM algorithm," Journal of Time Series Analysis, vol. 3, no. 4, pp. 253-264, 1982.

[18] N. de Freitas, M. Niranjan, A. H. Gee, and A. Doucet, "Global optimisation of neural network models via sequential sampling," in Advances in Neural Information Processing Systems, M. J. Kearns, S. A. Solla, and D. Cohn, Eds., vol. 11, MIT Press, 1999, pp. 410-416.

[19] J. Geweke, 'Bayesian inference in econometric models using Monte Carlo integration," Econometrica, vol. 24, pp. 1317-1399, 1989.

[20] G. Kitagawa, "Monte Carlo filter and smoother for non-Gaussian nonlinear state space models," Journal of Computational and Graphical Statistics, vol. 5, pp. 1-25, 1996.

[21] N. de Freitas, "Rao-Blackwellised particle fi ltering for fault diagnosis," in IEEE Aerospace Conference, 2001.

[22] C. Andrieu, A. Doucet, and E. Punskaya, 'Sequential Monte Carlo methods for optimal filtering," in Sequential Monte Carlo Methods in Practice, A. Doucet, N. de Freitas, and N. J. Gordon, Eds. SpringerVerlag, 2001.

[23] M. K. Pitt and N. Shephard, 'Filtering via simulation: Auxiliary particle fi lters," Journal of the American Statistical Association, vol. 94, no. 446, pp. 590-599, 1999.

[24] L. Ljung, System Identification: Theory for the User. Prentice-Hall, 1987.

[25] R. Washington, "On-board real-time state and fault identification for rovers," in IEEE International Conference on Robotics and Automation, 2000.

[26] R. Morales-Menendez, N. de Freitas, and D. Poole, 'Estimation and control of industrial processes with particle fi lters," in American Control Conference, 2003.

[27] C. Andrieu, M. Davy, and A. Doucet, "Effi cient particle fi ltering for jump markov systems," 2003, to appear in IEEE Transactions on Signal Processing.

[28] F. Hutter and R. Dearden, 'Effi cient on-line fault diagnosis for nonlinear systems," in Proceedings of the 7th International Symposium on Artificial Intelligence, Robotics and Automation in Space, 2003.

[29] _ - "The gaussian particle fi lter for diagnosis of non-linear systems," in Under Review for the 14th International Workshop on Principles of Diagnosis, 2003. 